This item was submitted to Loughborough's Research Repository by the author.

Items in Figshare are protected by copyright, with all rights reserved, unless otherwise indicated.

\title{
Simulating radiation damage in Ga stabilised $\delta-\mathrm{Pu}$
}

PLEASE CITE THE PUBLISHED VERSION

http://dx.doi.org/10.1016/j.nimb.2011.07.005

PUBLISHER

(c) Elsevier

VERSION

AM (Accepted Manuscript)

LICENCE

CC BY-NC-ND 4.0

REPOSITORY RECORD

Robinson, Marc, Steven D. Kenny, Roger Smith, and M.T. Storr. 2019. "Simulating Radiation Damage in Ga Stabilised $\Delta$-pu". figshare. https://hdl.handle.net/2134/11624. 
This item was submitted to Loughborough's Institutional Repository (https://dspace.lboro.ac.uk/) by the author and is made available under the following Creative Commons Licence conditions.

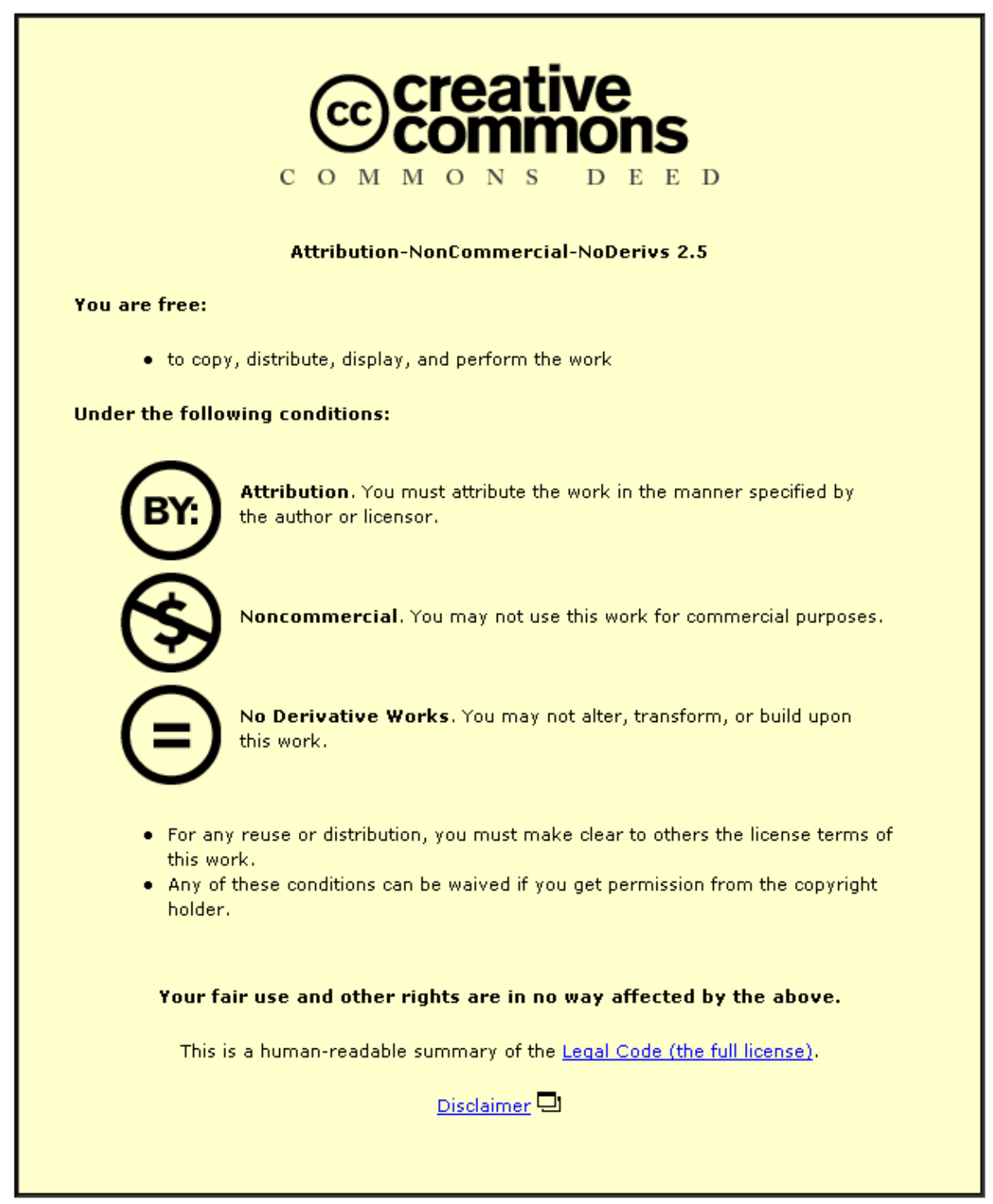

For the full text of this licence, please go to: http://creativecommons.org/licenses/by-nc-nd/2.5/ 


\title{
Simulating Radiation Damage in Ga stabilised $\delta$-Pu
}

\author{
M. Robinson, S.D. Kenny, R. Smith \\ Department of Mathematical Sciences, \\ Loughborough University, Loughborough, \\ LE11 3TU, UK \\ M.T. Storr \\ Atomic Weapons Establishment, \\ Aldermaston, Reading, \\ $R G 74 P R, U K$
}

\begin{abstract}
Radiation events in Ga stablised $\delta$-Pu are investigated by means of Molecular Dynamics simulations. $\mathrm{Pu} 5$ at. \% Ga is considered using the Modified Embedded Atom Method to govern the atomic interactions. Cascades were initiated with Primary Knock-on Atom (PKA) energies in the range of 0.4 to $10 \mathrm{keV}$, with trajectories deduced through comprehensive sampling of a representative set of directions, combined with different Ga atomic positions. The displacement threshold energy, $E_{d}$, for $\mathrm{Pu}$ and $\mathrm{Ga}$ atoms was also determined through similar extensive studies to aid the understanding and interpretation of the cascade results.

Values of $E_{d}$ between 5 and $40 \mathrm{eV}$ were determined for $\mathrm{Pu}$, with Ga PKAs requiring generally more energy to create a defect with $E_{d}$ between 8 and $70 \mathrm{eV}$. Low energy collision cascades, initiated with energies in the range of 0.4 to $1 \mathrm{keV}$, show that the cascades form in a similar manner to other fcc metals with a vacancy rich zone surrounded by isolated interstitial defects. A feature of these cascades is that the displaced Ga atoms return to lattice sites during the ballistic phase, leading to a lack of Ga-type residual defects. Higher energy cascades show similar features but with the development of an amorphous region at the cascade core of around $5 \mathrm{~nm}$ diameter at $5 \mathrm{keV}$. Quantitatively, the residual number of defects found shows no distinct variation to that for previous work on pure $\mathrm{Pu}$, suggesting the inclusion of Ga does not significantly effect the susceptibility or resistance of Pu to initial
\end{abstract}


cascade development.

\section{Introduction}

Due to its application in the nuclear industry, understanding the physical nature of $\mathrm{Pu}, \mathrm{a}$ highly complex material, is of increasing importance. However, experimentation is expensive and difficult due to the highly reactive and radioactive nature of $\mathrm{Pu}$. As a result, computer simulation has become a propitious technique to use for scientific analysis in this area.

The ageing of plutonium is a key process that affects its every use. This phenomena, inherent in all radioactive materials, generates issues with otherwise trivial topics, such as storage and handling. The continual atomic level radiation events lead to swelling and degradation which may induce macroscopic changes in structural properties. In $\mathrm{Pu}$ specifically, these events are initiated through $\alpha$-decay, generating a high energy He nucleus and an 85 $\mathrm{keV}$ recoiled uranium atom.

Plutonium is usually formed and stored in the fcc ( $\delta$-phase), favourable due to the relative structural integrity and manageability of the material compared to other phases. However, at room temperature $\delta$-Pu is thermodynamically unstable and stability has to be induced through a low percentage substitution of a 'delta-gen', commonly gallium [1]. However, the positive physical effects of alloying $\mathrm{Pu}$ with $\mathrm{Ga}$ consequently increase the complexity of modelling the material.

Previous work on modelling pure plutonium resulted in some reasonable success when employing the Modified Embedded Atom Method (MEAM) [2-5] to describe the atomic interactions. These studies indicate the ability of the MEAM to capture the complex phase diagram of plutonium as well as accurately determining various bulk properties. Valone et al. calculated defect formation energies which highlighted the mono-vacancy as requiring the least energy to form at around $1 \mathrm{eV}$ and interstitials favouring the $\langle 100\rangle$ orientation. Additionally, this work investigated displacement threshold energies $\left(E_{d}\right)$ giving a minimum value of around $10 \mathrm{eV}$, significantly lower than other fcc metals $(\mathrm{Ag}-25 \mathrm{eV}, \mathrm{Ni}-23 \mathrm{eV}$, $\mathrm{Cu}-19 \mathrm{eV})[6]$. Defect formation energies of a larger range of defect clusters were also calculated by Berlu et al. [7] whose findings supported the earlier work of Valone. Long 
time scale dynamics studies into vacancy migration by Uberuaga et al. [8,9] indicate the complexity of the MEAM potential energy surface when calculating energy barriers and the high sensitivity of the dynamics through only small changes in potential parameters. Kubota et al. [3] carried out MD simulations in $\delta$-Pu, with results suggesting that cascades evolved through an amorphous, glass-like structure with recovery times longer than the normal MD time frame. This feature was also reported through the MD simulations of Berlu et al.[10] and hybrid Monte-Carlo and MD simulations of Dremov et al.[11] which incorporated electron-phonon coupling. These findings agree well with the results from the precursor to this study [12], where cascades into pure $\mathrm{Pu}$ was investigated using the MEAM. This work found the lowest value of $E_{d}$ to be around $6 \mathrm{eV}$ in trajectories along the less close-packed directions, showing good agreement with other studies [13].

Investigations into the $\mathrm{Pu}-\mathrm{Ga}$ alloy have been carried out through adaptations and extensions to the MEAM [14]. These changes were developed through the incorporation of both the pure $\mathrm{Pu}$ scheme and a pure Ga MEAM implementation [15] developed by Baskes et al.. This development allowed for investigations into the properties of the alloy as the Ga concentration was varied, suggesting, amongst other things, that increasing Ga concentration up to a $25 \%$ limit, consistently lowered the total energy of the system. This finding was supported by further studies into Ga ordering [16], which indicated that Ga-Ga 1st nearest neighbour bonds are unfavourable, with the $25 \%$ Ga concentration limit representing the point at which these bonds are forced to form, dramatically increasing the total energy. Work including both experiment and simulation of $\mathrm{Ga}$ stabilised $\delta$ - $\mathrm{Pu}$, found that $10 \mathrm{keV}$ cascades would produce around 4500 Frenkel pairs during a ballistic phase of 4 ps, with 1700 pairs remaining after 0.1 ns [13]. Both this study and the work of Dremov [11], again reported the development of an amorphous core during cascade development, findings similar to those into pure $\mathrm{Pu}$.

The aim of this study is to investigate radiation events through the simulation of collision cascades. As most previous work examined a small number of cascades, this work adopts a systematic approach to analysis, obtaining good statistics and maximising the reliability of findings. Over 600 separate collision cascades are considered in the investigation from 
primary knock-on atoms (PKAs) directed over a representative region of the crystal. The number and types of interstitials and vacancies produced by the cascades are determined and analysed together with the structure of the cascades for a range of PKA energies between 0.4 and $10 \mathrm{keV}$. To accompany cascade simulations and to study defect production mechanisms, displacement threshold energies $\left(E_{d}\right)$ have also been calculated for both $\mathrm{Pu}$ and Ga PKAs.

\section{Methodology}

Radiation damage in Ga-stabilised $\delta$-Pu has been modelled by the use of molecular dynamics (MD), using an empirical potential to describe the interatomic interactions. Spatial decomposition parallelisation has been employed to enable large scale, atomic-level investigations for modelling collision cascades. The atomic interactions are governed by the MEAM [14], where the energy per atom $E_{i}$, is given by

$$
E_{i}=F_{i}(\rho)+\frac{1}{2} \sum_{j} \phi\left(r_{i j}\right)
$$

where $F_{i}(\rho)$ represents the embedding energy of atom $i$, which is deduced from the specific geometry of the local environment and $\phi\left(r_{i j}\right)$ is a pair potential chosen so as to give the correct equation of state. All MD simulations have been carried out with the parameters published by Baskes [14]. Close range interactions are modelled with the Ziegler-BiersackLittmark (ZBL) potential [17] to enable an accurate calculation of the energies in this range. The MEAM is splined to the ZBL potential using an exponential function which matches the function value, first and second derivatives at both spline points, ensuring a smooth transition. The spline is implemented between 0.7-1.8 $\AA$ for $\mathrm{Pu}-\mathrm{Pu}$ interactions, 0.7-1.2 $\AA$ for Ga-Ga and 0.2-0.4 $\AA$ for Pu-Ga. A cutoff of $4.5 \AA$ was employed, with potential contributions smoothed to zero at this point.

Radiation damage simulations have been initiated by a primary knock-on atom (PKA) with a given kinetic energy in a particular direction. Simulations have been performed with PKA energies in the range $400 \mathrm{eV}$ to $10 \mathrm{keV}$, to generate a comprehensive picture of each stage of the radiation process. This is lower than the initial kinetic energy generated during a radiation event, so can be thought of as a collision from a secondary recoiled atom. 
System sizes modelled range between 30,000 and 1.1 M atoms, dependent on PKA energy, with periodic boundary conditions employed in all directions. All collision cascade simulations have been performed at $300 \mathrm{~K}$, with systems initially relaxed using the conjugate gradient method [18] with thermal equilibrium achieved using the Nose-Hoover thermostat [19]. The direction of the PKA trajectory is chosen to be sampled within a reduced set of directions, see Figure 1 (a) due to the innate symmetry of the fcc cell. Simulations are performed that sample a range of directions within this reduced zone so as to maximise the generality of the results. Figure 1 (b) shows the directions employed for the $400 \mathrm{eV}$ and 1 $\mathrm{keV}$ cascades and the threshold calculations. The number of PKA directions used combined with 5 different lattice arrangements of the substitutional Ga means that relatively good statistics have been obtained.

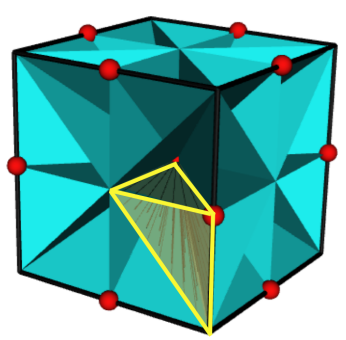

(a)

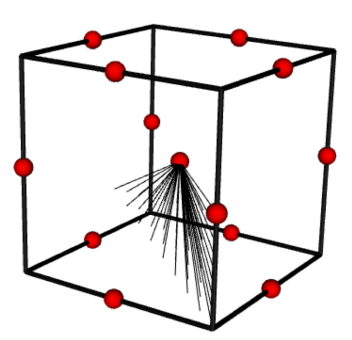

(b)

Figure 1: (a) The irreducible symmetry zone for the fcc lattice. (b) The 45 directions utilised for the displacement threshold energy calculations and the $400 \mathrm{eV}$ and $1 \mathrm{keV}$ collision cascades.

Cascades have been carried out using 5 atomic \% substitutional $\mathrm{Ga}(\mathrm{Pu} 5$ at. \% Ga) as this lies within the stable concentration bounds of between 2 and $9 \%$ at ambient temperature [1]. Cascades were initiated with PKAs having energies of $400 \mathrm{eV}, 1 \mathrm{keV}, 2 \mathrm{keV}$, $5 \mathrm{keV}$ and $10 \mathrm{keV}$. For the 2, 5 and $10 \mathrm{keV}$ PKA simulations, 60 trajectories each were investigated consisting of 12 directions over the symmetry zone and 5 different $\mathrm{Pu} 5$ at. \% Ga arrangements. For the $400 \mathrm{eV}$ and $1 \mathrm{keV}$ cascades, again 5 different $\mathrm{Pu} 5$ at. $\% \mathrm{Ga}$ arrangements were used but the number of PKA directions was increased to 45, see Figure 1. Results are analysed through the calculation of the number of defects (vacancy and interstitial pairs) produced, with colour denoting species, (i.e $\mathrm{Pu}=$ red and $\mathrm{Ga}=$ blue) to 
give a clear indication of damage. Any sites that have become occupied by a different specie are shown as wireframe cube (coloured via the specie of the initial site), containing the new occupying atom. This 'defect' will be referred to as an anti-site.

To aid the cascade analysis, a tool has been developed that gives an indication of cascade size and structure as well allowing for categorisation of outlying defect clusters. This allows the detection of single intense cascade cores to multiple cores dispersed through sub-cascades branching. In addition to this, there is a defect classification scheme which allows isolated defects and small defect clusters, separated from the main cascade volume to be analysed. The technique adopted to carry out this analysis can be broken down into two main processes; domain decomposition and volume triangulation. The first of these is relatively trivial and is implemented through recursively searching the neighbour list of each defect and assigning the appropriate region to every listed defect. This is carried out using a cut-off distance (3.5 $\AA$ ) which acts as a skin around each region. Once the defects are divided into regions, the second process of volume triangulation can be carried out. There exists various algorithms concerning triangulation of 3D point clouds, such as Delauny triangulation [20], but at the root of each is a Convex Hull technique [21], which is adopted here. This method recursively creates convex polyhedra whilst continuously testing that all points lie inside the current bounding surface. This results in a triangulated surface which is used in conjunction with Gauss' Theorem concerning a closed surface to calculate the enclosed volume. An example of how this technique progresses during cascade analysis can be found in Figure 14. Using this methodology allows the cascade core volume to be analysed as a function of simulation time.

A by-product from the implementation of the volume analysis and region decomposition tool is the ability to capture isolated defect clusters. These clusters, once removed from the bulk cascade core, can be analysed to give a distinct picture of probable remaining defects as well as giving direct insight into how the cascade has evolved. To ensure that all defect categories are captured, analysis is carried out for both specie as well as mixed defect clusters.

In order to ascertain the radiation tolerance of Ga-stabilised $\mathrm{Pu}$, threshold displacement 
energies $\left(E_{d}\right)$ have been calculated. The complexities outlined in the cascade simulation techniques due to the changes in Ga configuration mean that calculating valid values of $E_{d}$ is a time consuming task. To gain representative values, a large number of possible Ga arrangements must be investigated along with the complete sampling of the irreducible volume. To make this possible, parallelisation is again employed but in a different manner to that implemented in the MD program. Instead, a series of serial MD jobs are distributed to up to 40 processors, each calculating a PKA trajectory in a certain $\mathrm{Pu} 5$ at. \% Ga lattice. The automated processes consist of a bisection search algorithm that at each step runs an MD collision cascade for 4 ps. The PKA energy is appropriately varied until the bracketted region is less that $0.5 \mathrm{eV}$. This procedure was carried out for both $\mathrm{Pu}$ and $\mathrm{Ga}$ as the PKA, using 5 different equilibrated $\mathrm{Pu} 5$ at. \% Ga configurations, with 8 different PKA locations to generate the 40 random local configurations. Combining this with the 45 separate PKA directions as used in the low energy cascades (Figure 1), results in 3600 values of $E_{d}$.

To enable cascade simulations into Ga-stabilised $\mathrm{Pu}$, the ordering of the Ga within the $\mathrm{Pu}$ lattice needs to be considered. Due to the inherent instability of $\mathrm{Pu}$ and the complexity of the potential energy surface generated through fitting the MEAM to such an extreme phase diagram, calculation of Ga ordering using MD methods is not pursued. Other studies into Ga ordering [16] via a Monte Carlo approach indicated that lattices set up with at least 2nd nearest neighbour $(2 \mathrm{NN})$ Ga spacing gave the lowest energy structure. A novel method of doing this is to create a $\mathrm{Pu}_{3} \mathrm{Ga}$ lattice ( $\mathrm{L}_{2}$ space group), with Ga located at the corner sites of the fcc cell, giving a maximum of $25 \%$ Ga. The required concentration can then be achieved through random removal of Ga atoms. However, although this method removes the high energy Ga $1 \mathrm{NN}$ bonds, it results in a biased configuration, as the Ga-Ga radial distribution function (RDF) shows in Figure 2(b). This occurs as a result of the placement of $\mathrm{Ga}$ on $2 \mathrm{NN}$ sites in the original $\mathrm{Pu}_{3} \mathrm{Ga}$ lattice, preventing the possibility for Ga in $3 \mathrm{NN}$ and $5 \mathrm{NN}$ positions and promoting $2 \mathrm{NNs}$ and $4 \mathrm{NNs}$. This would not be an issue if the desired percentage of Ga was $25 \%$, as this is the only configuration possible which would have no Ga 1NNs. However, as the Ga is a stabiliser and is only included in small percentages, there is a clear possibility for Ga at $3 \mathrm{NN}$ and $5 \mathrm{NN}$ sites. To circumvent this 
issue, a brute force method was employed that randomly substituted a $\mathrm{Pu}$ atom for $\mathrm{Ga}$ at the same time as ensuring no Ga 1NNs. This results in the RDF in Figure 2(c) showing now a $3 \mathrm{NN}$ component. To guarantee the Ga arrangement within the lattice is a minimum energy configuration, a Monte Carlo technique is employed, similar to that employed by Baskes et al. [16]. At each iteration a random $\mathrm{Ga}$ is chosen and switched with a $\mathrm{Pu} 1 \mathrm{NN}$ and the new potential energy calculated. Acceptance occurs if the generated probability is lower than :

$$
P_{\text {Acceptance }}=e^{-\frac{\delta E}{k_{b} T}}
$$

where $T$ is the simulated temperature, which is reduced every 1000 steps of the MC and $\delta E$ is the difference in energy from the previous configuration. The resultant RDF after the $\mathrm{MC}$ and relaxation is shown in Figure 2(d). As expected the Ga 1NN are almost completely removed, but there is an increase in $2 \mathrm{NN}$ and $3 \mathrm{NN}$ with a corresponding reduction in $4 \mathrm{NN}$ and $6 \mathrm{NN}$. These results are in line with previous work [16]. Although this method produces low energy configurations, the energy change from a random Ga arrangement (corresponding to the rdf in Figure 2(a)) is only around $5 \times 10^{-3} \mathrm{eV} /$ atom. This is due to the small percentage of $\mathrm{Ga}$ in the system which means that in the random Ga setup, the number of Ga-Ga 1NN bonds formed is low enough as to not to significantly increase the total potential energy of the system. As the MC based lattice initialisation is considerably more computationally expensive in comparison to the $\mathrm{Pu}_{3} \mathrm{Ga}$ setup, both will be examined for the displacement threshold energy calculations and initial collision cascade simulations.

\section{Results}

\subsection{Displacement threshold energies}

Displacement threshold energies have been calculated for both specie of atom, allowing for direct comparisons not only within the $\mathrm{Pu} 5$ at. \% Ga system but against the previous pure $\mathrm{Pu}$ work. The full set of $3600 E_{d}$ calculations were computed for each Ga arrangement whose RDFs are shown in Figure 2 (b)-(d) and the results indicated no distinct differences. This suggests that $E_{d}$ is not significantly effected by the Ga-Ga bond order past the 1NN 


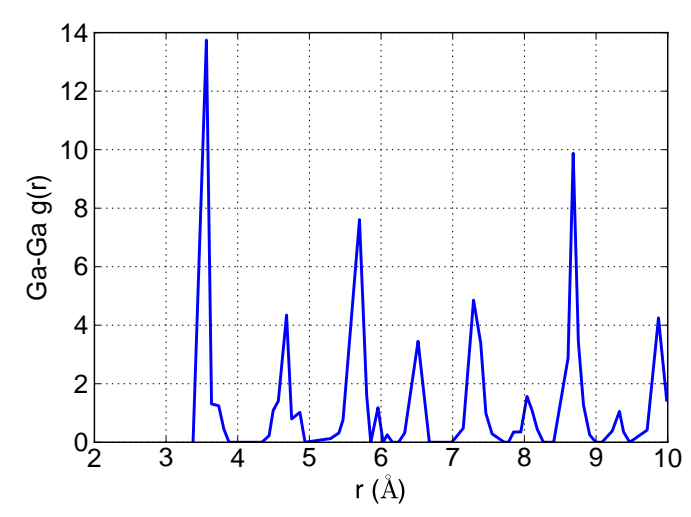

(a)

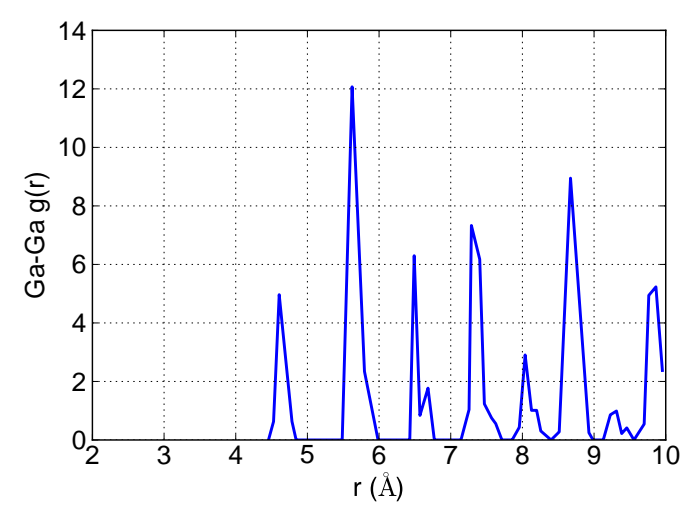

(c)

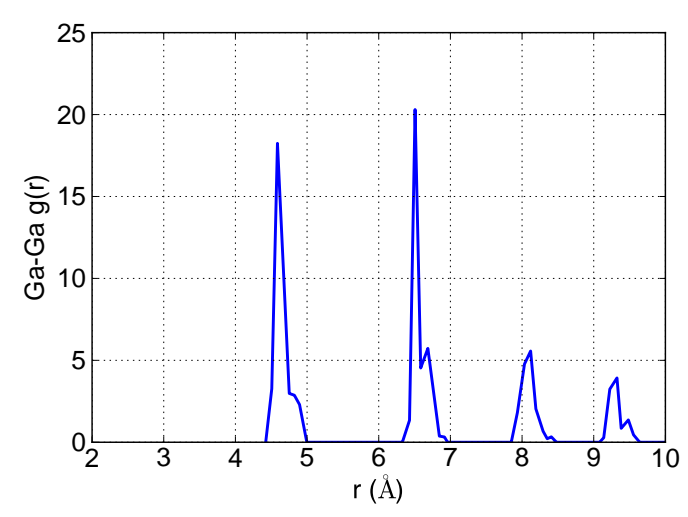

(b)

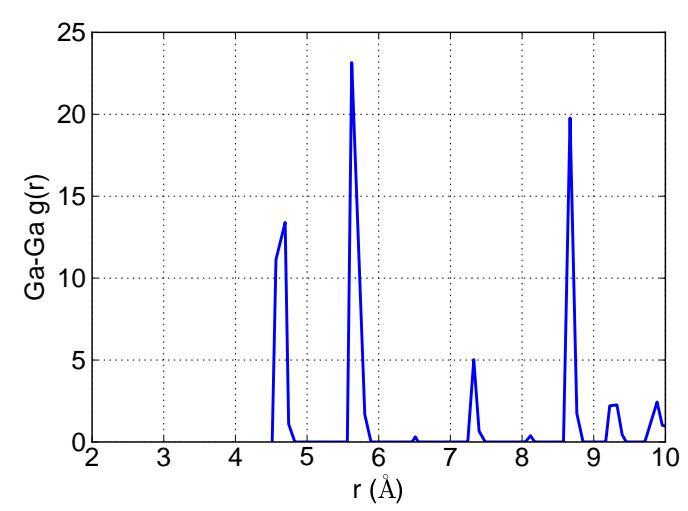

(d)

Figure 2: Radial distribution functions of Ga-Ga bond lengths for various relaxed $\mathrm{Pu} 5$ at. \% Ga configurations. (a) Purely random Ga substitutions, (b) $\mathrm{Pu}_{3} \mathrm{Ga}$ initial setup and random removal of Ga atoms, (c) Random substitutions of Ga given each has no Ga 1NN, (d) Monte Carlo simulation result after 20000 steps.

position, suggesting it is only important to remove $1 \mathrm{NN}$ Ga bond when initialising the $\mathrm{Pu}$ 5 at. $\%$ Ga lattices.

The distribution of $E_{d}$ values calculated for both specie are shown as in Figure 4. What is initially clear from these results is that the Ga PKA requires significantly more energy to create a defect than the Pu PKA in the majority of simulations. The minimum value of $E_{d}$ in $\mathrm{Pu} 5$ at. \% $\mathrm{Ga}$ is around 5-6 eV, calculated for the Pu PKA, with the lowest value of $E_{d}$ calculated for the Ga PKA is higher at around 9-10 eV. This increase is expected at low kinetic energies due to the reduced transfer of momentum from the lighter Ga PKA to the 
heavier $\mathrm{Pu}$ neighbours during the initial collision. The minimum value of $E_{d}$ found for the $\mathrm{Pu} \mathrm{PKA}$ relates well to that found in pure $\mathrm{Pu}[12]$. This is due to the $\mathrm{Pu}$ rich environments local to the PKA site which, at low PKA energies, will behave as observed in pure Pu. In contrast to this similarity, the maximum observed values of $E_{d}$ are much larger than that found in pure $\mathrm{Pu}$, with the $\mathrm{Pu} \mathrm{PKA}$ requiring $40 \mathrm{eV}$ and $\mathrm{Ga} \mathrm{PKA}$ requiring up to $70 \mathrm{eV}$ to create a defect. As a consequence of these extremes, a large range of possible $E_{d}$ values are observed. To understand how the introduction of $\mathrm{Ga}$ is causing this distribution of $E_{d}$ values, the dependence of $E_{d}$ on PKA trajectory is analysed. Figures 3(a) and 3(c) show the resultant values of $E_{d}$ for each specie, with trajectories representing an average across the 40 lattice configurations. This data is then extrapolated through symmetry to form Figures $3(\mathrm{~b})$ and $3(\mathrm{~d})$ to give a more intuitive picture.

What is initially clear from Figure 3 is that the Ga PKA, as indicated by the distribution of $E_{d}$, requires significantly more energy to create a defect in almost all directions in comparison to $\mathrm{Pu}$. An extreme case of this is in the $\langle 100\rangle$ trajectory where $\mathrm{Ga}(\sim 40 \mathrm{eV})$ requires around 4 times more energy than its $\mathrm{Pu}$ counterpart $(\sim 10 \mathrm{eV})$. In contrast, the $\langle 110\rangle$ crystallographic direction results in a similar values of $E_{d}$ for the Ga PKA yet as this is the 1st nearest neighbour, close packed direction, it might have been assumed this would require more energy to create a defect. It is therefore clear that a simple explanation based upon binary collision sequences along these trajectories is not adequate. Looking into the specific simulations in more depth reveals an interesting mechanism that aids in explaining this increased value of $E_{d}$ along the $\langle 100\rangle$ as well as the $\langle 111\rangle$ direction. Figure 5 shows the mechanism observed during the $\mathrm{Ga}\langle 100\rangle$ simulation as both a schematic representation as well as an actual MD result. What this mechanism shows is that the Ga initially impacts 4 1NNs (indicated by ' $\mathrm{C}$ ' in Figure 5), before going on to impact the first atom along the $\langle 100\rangle$. Displacing these atoms requires substantial amounts of energy, as these 1NNs are guaranteed to be the heavier $\mathrm{Pu}$ due to the removal of all higher energy Ga-Ga 1NN bonds as discussed previously. There is some variation in the second collision, as the Ga's $2 \mathrm{NN}$ may be either $\mathrm{Pu}$ or $\mathrm{Ga}$. The highest value of $E_{d}$ are found when the $2 \mathrm{NN}$ of the $\mathrm{Ga}$ is $\mathrm{Pu}$, as in Figure 5. What makes the values of $E_{d}$ along the $\langle 100\rangle$ direction higher still are the 
number of collisions necessary before the first defect is created, with the proportion of $\mathrm{Pu}$ atoms involved in these collisions linking directly to the increase in $E_{d}$. The defect is finally created in this mechanism through a displacement in $\langle 110\rangle$, which was also found to give the lowest value of $E_{d}$ for both Ga and Pu PKAs. A similar reasoning for the increase in Ga $E_{d}$ can be applied to the $\langle 111\rangle$ trajectory as shown in Figure 6. This mechanism involves the Ga atom initially causing deflections with $3 \mathrm{Pu} 1 \mathrm{NN}$ atoms that lie on a plane bisected by the PKA's path. This allows the Ga PKA to sit on one of the three now vacant lattice sites. Each of the deflected atoms go on to cause secondary collision sequences along the $\langle 110\rangle$ direction. This results in the final defect lying relatively far from the initial PKA site with the collision pathway lined with a series of intermediate replacements. In contrast to this, the $\mathrm{Pu}$ PKA mechanism consists simply of creating a local defect pair, along the initial $\langle 111\rangle$ direction, with the defect consisting of either the PKA or one of the 3 nearest neighbours previously mentioned. These differences in mechanism suggest numerous reasons for the variation in $E_{d}$, as well as highlighting possible features that may occur during the cascade simulations.

\subsection{Collision cascades}

As with the displacement threshold energy calculations, to highlight the dependency of the cascade on the initial Ga setup, the $1 \mathrm{keV}$ cascades were carried out using both the $\mathrm{Pu}_{3} \mathrm{Ga}$ methodology of setting up the lattice which gives the RDF shown in Figure 2(b) and the Monte-Carlo approach which results in the RDF in Figure 2(d). The resultant damage from these cascades indicated that the difference in both the number of peak and residual defects lies well within the statistical error. This suggests that, as with the values of $E_{d}$, there is no significant dependency on Ga-Ga bond order past 1NNs. For this reason and due to the time taken for the MC setup exponentially increasing with system size, all further cascade findings were carried out using the $\mathrm{Pu}_{3} \mathrm{Ga}$ based lattice initialisation.

Figure 7 shows the number of residual defects that originate along the different PKA directions for $400 \mathrm{eV}$ and $1 \mathrm{keV}$ cascades. This clearly shows no correlation with the calculated values of $E_{d}$ along the same directions (Figure 3). This is due to numerous random varia- 


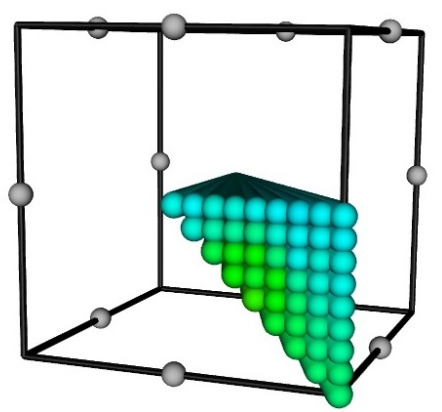

(a)

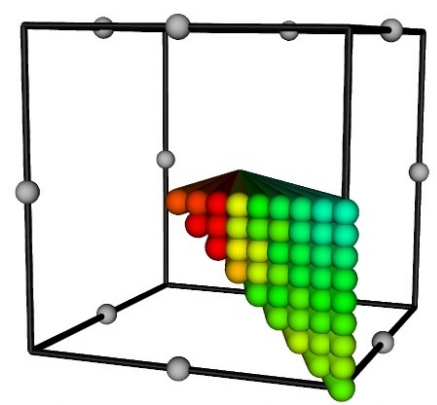

(c)

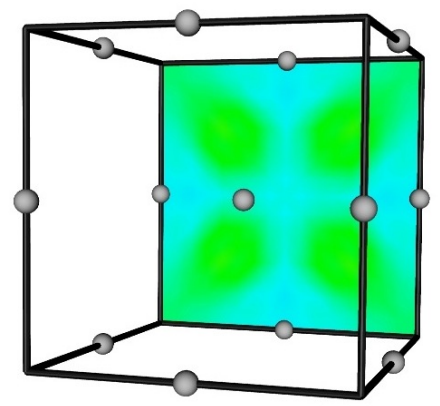

(b)

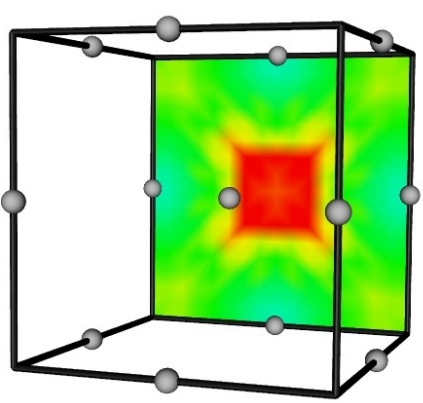

(d)

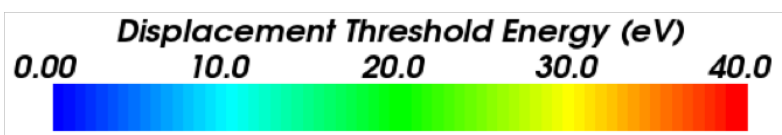

Figure 3: Average displacement threshold energies $\left(E_{d}\right)$ in $\mathrm{Pu} 5$ at. \% Ga. Images in (a) and (b) represent the plutonium values of $E_{d}$ with images in (c) and (d) representing gallium. Images (b) and (d) are constructed by extrapolating the $E_{d}$ data through symmetry.

tions in local environment such as thermal motion and Ga configuration. In addition, with the energy of the PKA now much larger than the even the maximum $E_{d}$, the mechanisms for creating defects would be highly complex such as the secondary collisions at the cascade core between atoms no longer in the fcc structure. Therefore, applying the reasoning behind the displacement threshold energy results to the cascade findings would not be adequate.

The variation in the number of residual defects as a function of time can be seen in Figure 8, with the maximum and minimum number of defects highlighted for the different PKA directions. These trajectories of maximum and minimum damage indicate a link between disorder and overall extent, with the reduction in point defects coming as a result of a larger affected volume. It is clear that this relationship is valid for low energy events, where no 


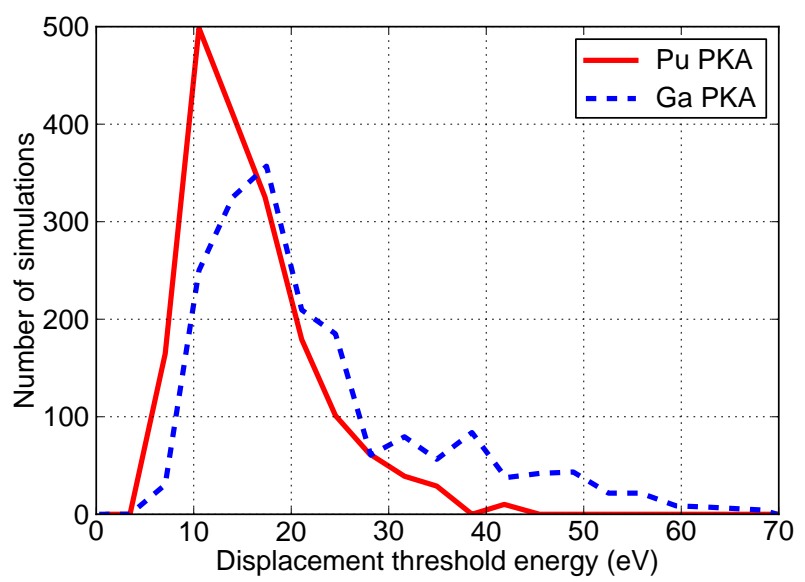

Figure 4: Distribution of displacement threshold energies $\left(E_{d}\right)$ in $\mathrm{Pu} 5$ at. $\%$ Ga for both $\mathrm{Pu}$ (shown as solid red) and Ga (shown as dashed blue) PKAs.

local melting occurs or complex defect structures such as dislocation loops are created.

One of the main features of the initial cascades is the lack of residual Ga interstitials. Further investigation, indicated that Ga interstitials created in the ballistic phase returned to either the initial Ga site or a close lattice site. This suggests that the transition barrier between Ga-type interstitials to pure Pu-type with the $\mathrm{Ga}$ in a lattice site is low. This behaviour is in contrast to the isolated $\mathrm{Pu}$ interstitials, which are more stable once formed.

Trends suggested in the low energy studies continued into the higher energy cascades, with the addition of a larger amorphous core region of around $5 \mathrm{~nm}$ in diameter at $5 \mathrm{keV}$ (Figure 9). Figure 10, that shows the RDF for the core region both before and after the collision cascade, indicates that no underlying structure is observed post cascade. This coupled with no distinct Ga segregation suggests this region is amorphous. This feature is well reported for pure Pu by Berlu et al.[10] and Dremov et al.[11] as well as in the precursor to this study [12]. The observation of the amorphous region is due to the melting of the cascade core, the result of concentrated heating during the first few ps of the cascade, a feature of cascades in metals initially simulated by Guinan et al. [22] and Diaz de la Rubia et al. [23].

A possible mechanism that could remove kinetic energy from the cascade is the creation 

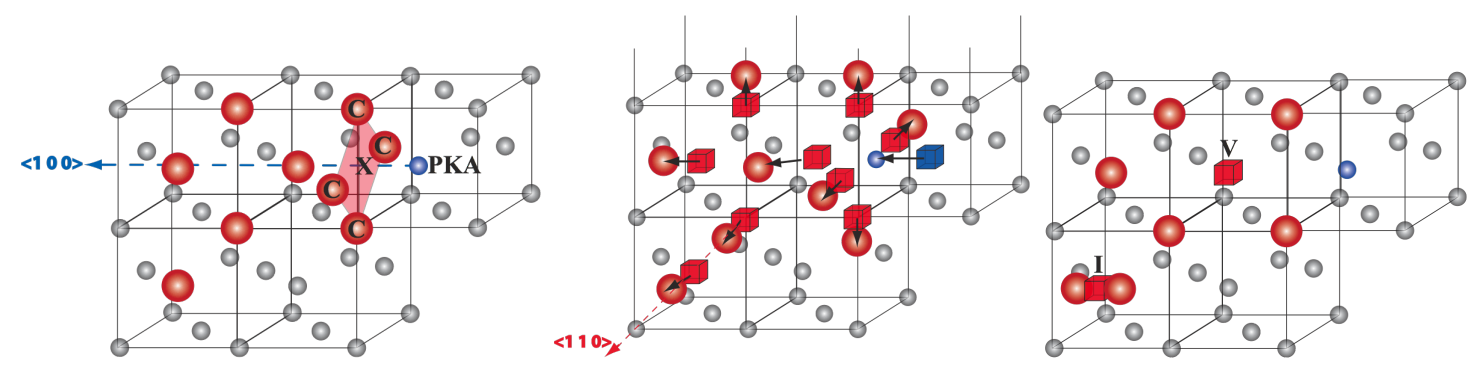

(a)
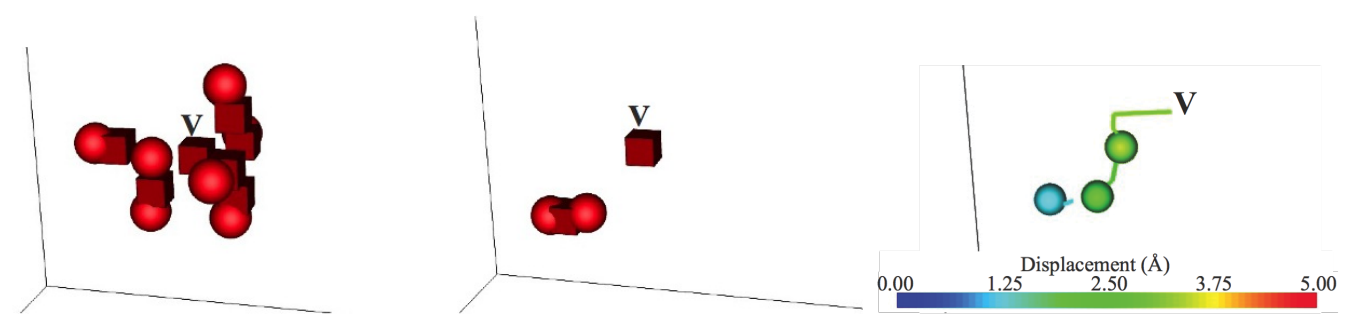

(b)

Figure 5: The displacement mechanism for a Ga atom along $\langle 100\rangle$ (Time passing left to right). a) Schematic representation where; ' $\mathrm{X}$ ' denotes the point of primary impact, ' $\mathrm{V}$ ' denotes vacancy left by the Ga PKA, 'C' denotes collisions with $1 \mathrm{NNs}$ and ' $\mathrm{I}$ ' denotes the residual interstitial. b) Results from the MD simulation filtered by point defects with the final image representing atomic displacements.

of Ga-Ga 1st nearest neighbours bonds. As the Ga-Ga 1NN bond is higher in energy and the $\mathrm{Pu} 5$ at. \% Ga lattice is initially constructed with no bonds of this type, any build up would indicate where the cascade energy is being spent. However, when the Ga bond ordering was studied, the change in Ga $1 \mathrm{NN}$ bonds was found to be negligible.

Another characteristic of higher energy cascades is the occurrence of sub-cascade formation, which becomes more prevalent as the PKA energy increases due, in part, to the reduction in collision cross-section. This results in separated, smaller regions of damage. The overall result is a reduction in the total number of defects produced in comparison to cascades with a single intense core. Sub-cascade formation becomes more frequent as PKA energies increase over $5 \mathrm{keV}$, with $10 \mathrm{keV}$ simulations indicating the formation of multiple sub-cascades.

As in the low energy studies, there are few Ga-type interstitial defects forming at higher energy. Figure 9 clearly shows this, with all of the various outlying defects and defect clusters being of type $\mathrm{Pu}$. Figure 11 shows anti-site defects which also indicates a large number of 

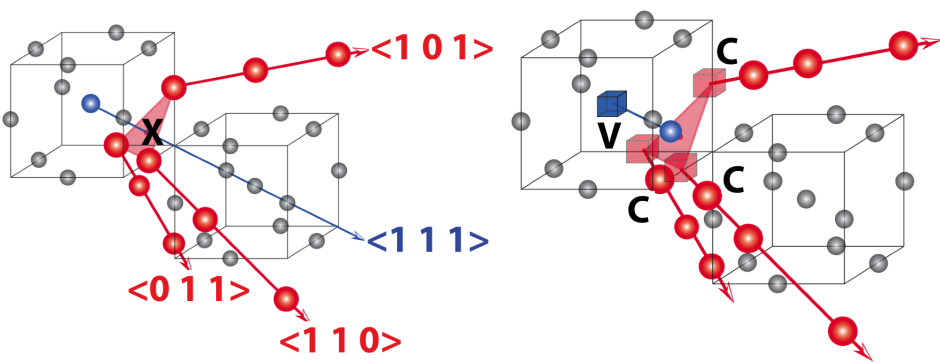

(a)
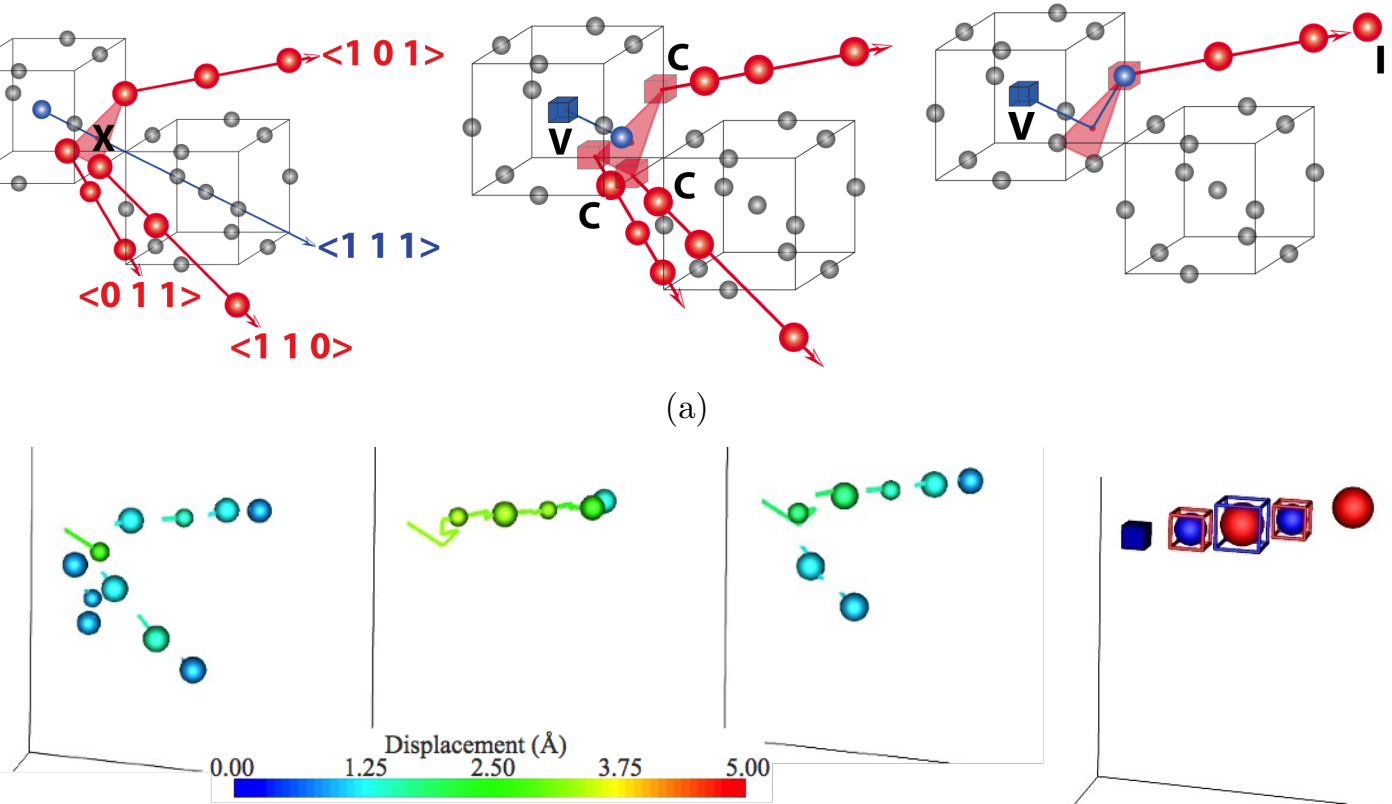

(b)

Figure 6: The displacement mechanism for a Ga atom along $\langle 111\rangle$ (Time passing left to right). a) Schematic representation where; ' $\mathrm{X}$ ' denotes the point of primary impact, ' $\mathrm{V}$ ' denotes vacancy left by the Ga PKA, 'C' denotes focussed collision sequences along $\langle 110\rangle$ and 'I' denotes the residual interstitial. b) Results from the MD simulation filtered by atomic displacement with the final image representing point defects.

Ga atoms located in initial $\mathrm{Pu}$ sites.

When comparing the overall defect production from the cascades in $\mathrm{Pu} 5$ at. \% Ga to previous work on pure $\mathrm{Pu}$ [12], as shown in Figure 12, it is clear that the inclusion of $5 \%$ Ga does not significantly alter the number of residual defects after the collisional part of the cascade. However, there is an increase in the average peak number of defects, suggesting a different underlying mechanism, induced by the presence of the Ga. Defect numbers were categorised by species, with Ga defects shown in Figure 13 which compares with the expected number of Ga defects taken as $5 \%$ of the total. Figure 13 clearly shows that the peak number of defects is as expected based on the stoichiometry.

As more than $90 \%$ of the total defects are concentrated in the highly disordered core region(s), a possible explanation for the increase at peak could be due to an increased volume of the defect rich regions. Volume analysis of the disordered core region has been carried out for all $5 \mathrm{keV}$ cascades. This data is shown in Table 2. The data shows an increase in 


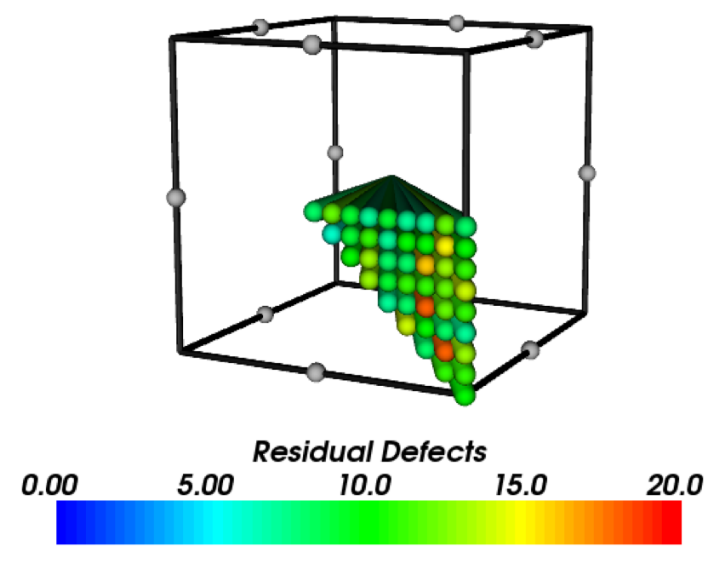

(a)
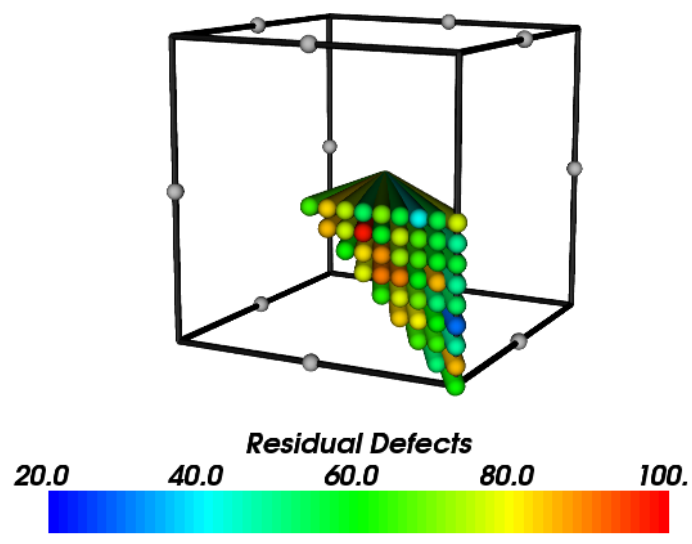

(b)

Figure 7: Average residual defects as a function of PKA trajectory in Pu 5 at. \% Ga for (a) $400 \mathrm{eV}$ and (b) $1 \mathrm{keV}$ cascades after $20 \mathrm{ps}$.

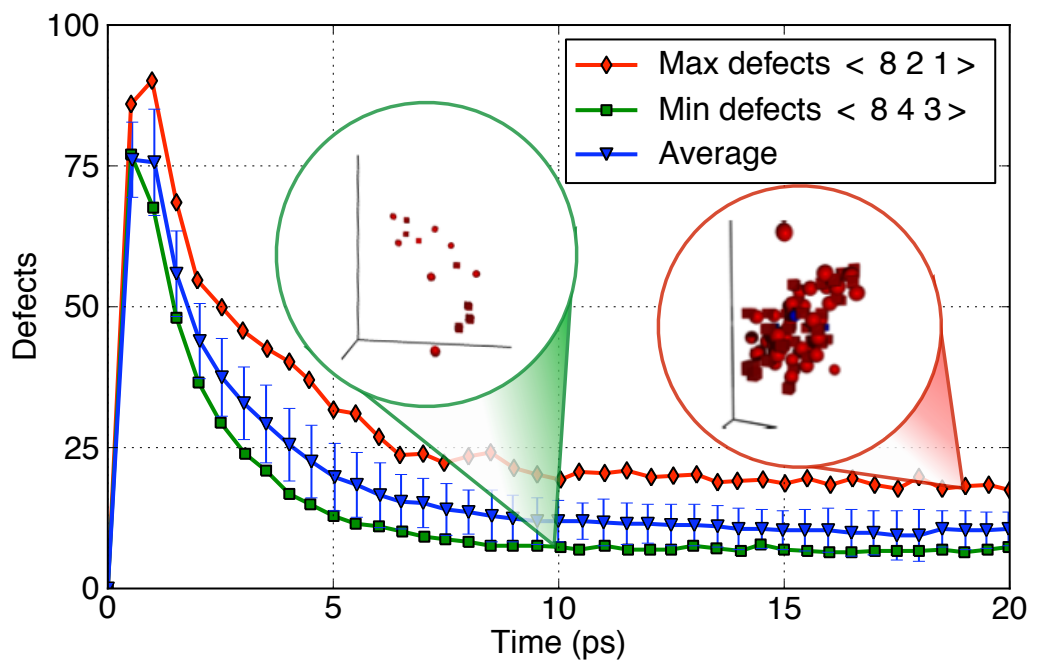

Figure 8: $400 \mathrm{eV}$ cascade defect evolution as a function of time in $\mathrm{Pu} 5$ at. \% Ga. Red and green plots highlight PKA trajectories which caused minimum and maximum damage respectively, with error bars representing one standard deviation. 


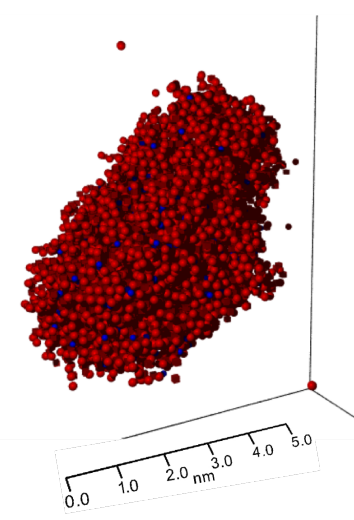

(a)

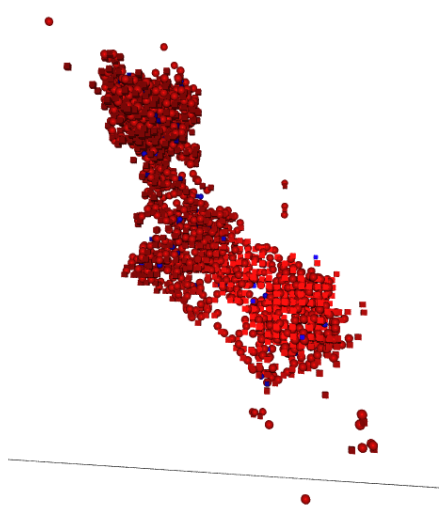

(b)

Figure 9: Residual defects from high energy cascades into Pu 5 at. \% Ga. (a) A typical $5 \mathrm{keV}$ cascade structure after $20 \mathrm{ps}$, highlights the amorphous zone of around $5 \mathrm{~nm}$ diameter at the cascade core. (b) At higher energies branching of the cascade leads to sub-cascade formation. The images indicate the generation of isolated $\mathrm{Pu}$ interstitials surrounding the core, with an absence of Ga-type defects.

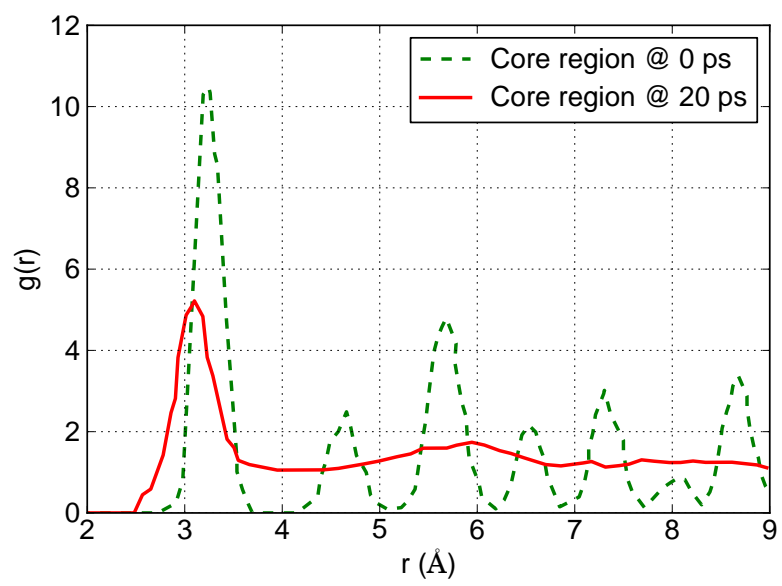

Figure 10: The RDF within the core region before and after a $10 \mathrm{keV}$ cascade. The dashed green plot is the initial distribution post thermalisation with the disordered region after 20 ps shown in solid red. 


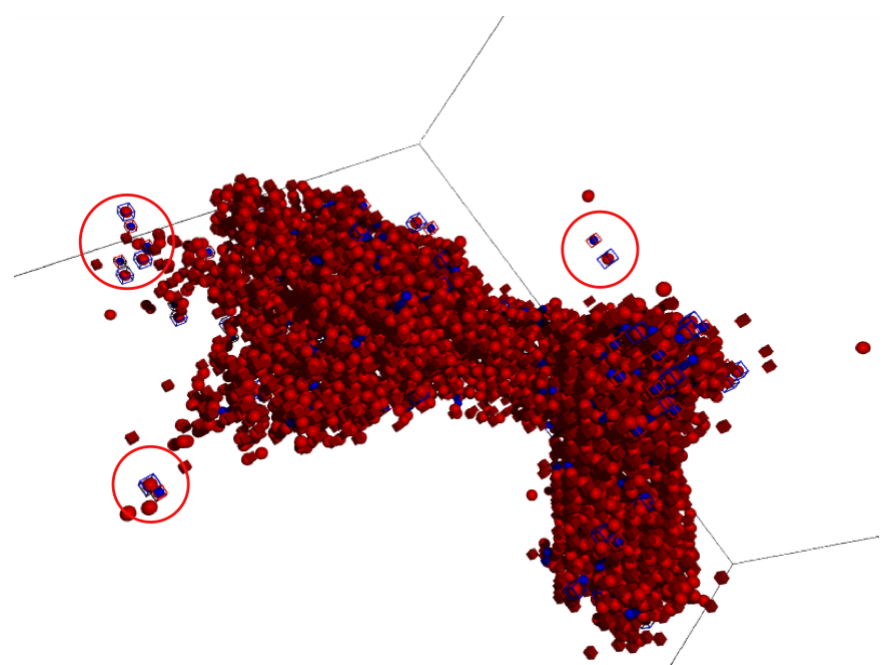

Figure 11: A $10 \mathrm{keV}$ cascade in $\mathrm{Pu} 5$ at. \% Ga, filtered by point defects. Red rings highlight displaced Ga atoms that have returned to initial $\mathrm{Pu}$ sites, resulting in no outlying $\mathrm{Ga}$ interstitials.

the peak number of defects of around $25 \%$ with the inclusion of the $5 \%$ Ga, but only a 14 $\%$ rise the volume of the core.

Cluster analysis outside the core region shows few Ga interstitials but many anti-site defects. This suggests that the lighter Ga atoms are being displaced during the initial high energy collisions, contributing to an increase the number of defects at peak, but soon return to lattice sites. The overall result is that a similar number of residual defects form in both pure $\mathrm{Pu}$ and $\mathrm{Pu} 5$ at. \% Ga. There is evidence for this from the mechanism observed during $\langle 111\rangle$ displacement threshold energy simulations, where the Ga once displaced returns promptly to a lattice site, temporally increasing the number of defects yet leaving only a single residual Frenkel pair.

Using the volume analysis method it is possible to study the defects that lie outside the central core region. These are shown for the $5 \mathrm{keV}$ cascades in Table 1 . This shows a large number of Ga antisites. If this number is broken down by species and separation, then most of the displaced Ga are lying in sites close to their initial positions.

To compare against other fcc metals, the number of defects as a function of PKA energy is fitted to the power law relationship: 


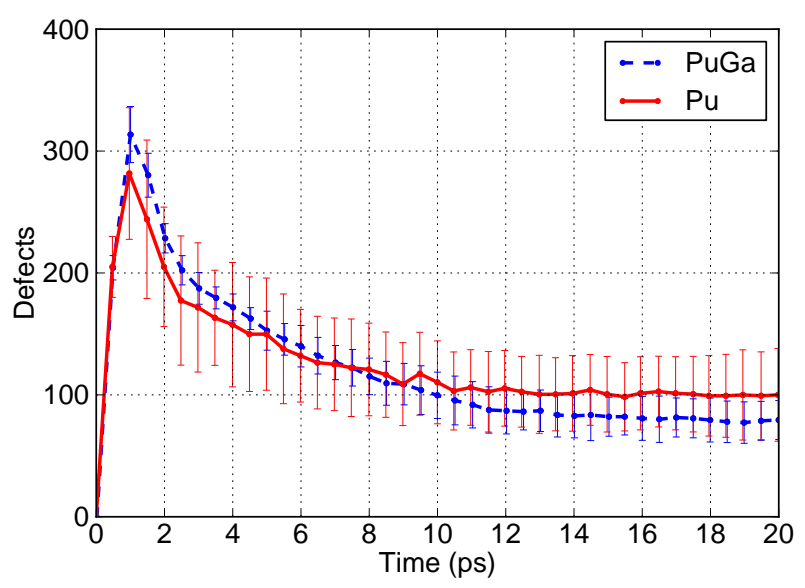

(a)

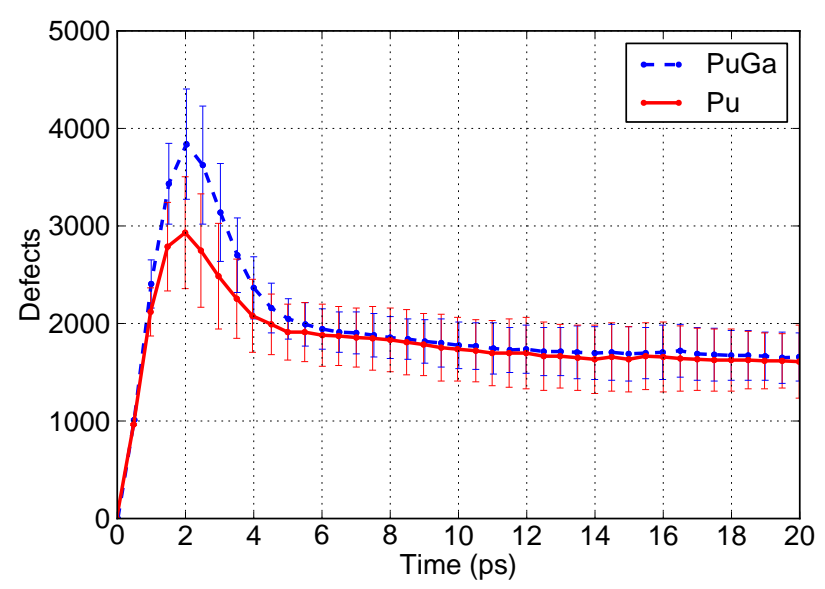

(b)

Figure 12: Comparisons of (a) $1 \mathrm{keV}$ and (b) $5 \mathrm{keV}$ defect production between the $\mathrm{Pu} 5$ at. \% Ga and pure $\mathrm{Pu}[12]$ systems. Error bars represent one standard deviation. 


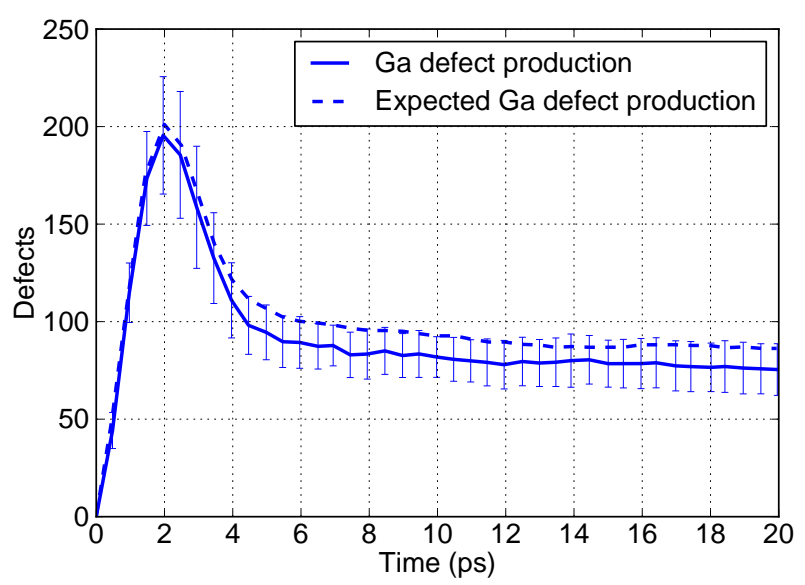

Figure 13: $5 \mathrm{keV}$ Average Ga defect production and expected number of defects (expected $=5 \%$ of total).

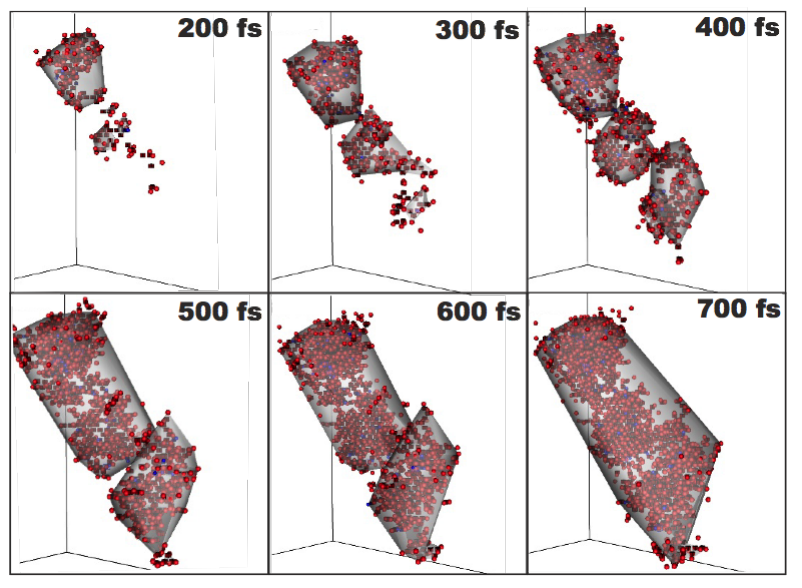

(a)

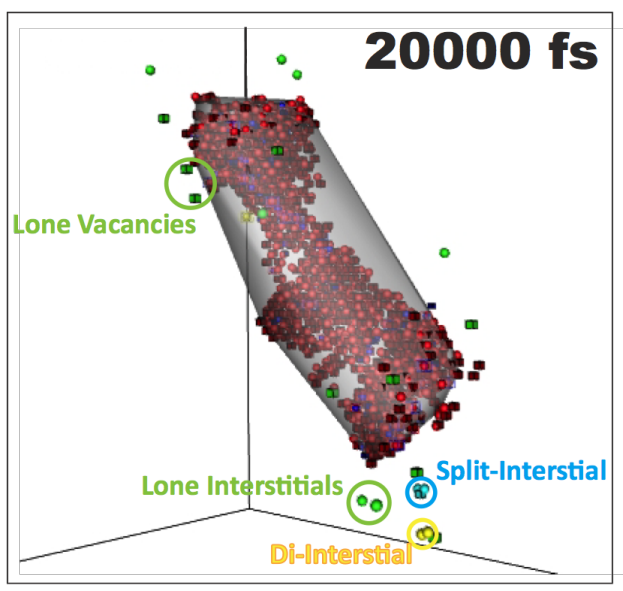

(b)

Figure 14: a) Volume bounding via 3D triangulation and defect domain decomposition with a neighbour cut-off of $3.5 \AA$, highlights how volumes merge as the cascade forms. b) Residual outlying defect clusters are classified to give an indication of remaining damage. 


\begin{tabular}{|c|c|c|c|c|}
\hline \multicolumn{5}{|c|}{$\begin{array}{c}\text { Pu } 5 \text { at. \% Ga } 5 \mathrm{keV} \\
\text { Cascades Defect Analysis }\end{array}$} \\
\hline & $\mathrm{Ga}$ & $\mathrm{Pu}$ & Mixed & Total \\
\hline \multicolumn{5}{|c|}{ Constituents } \\
\hline Vacancies & 1 & 298 & $\mathrm{~N} / \mathrm{A}$ & 299 \\
\hline Interstials & 2 & 303 & $\mathrm{~N} / \mathrm{A}$ & 305 \\
\hline Anti-sites & 123 & 131 & $\mathrm{~N} / \mathrm{A}$ & 254 \\
\hline \multicolumn{5}{|c|}{ Defect Categories } \\
\hline Lone interstials & 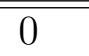 & 246 & $\mathrm{~N} / \mathrm{A}$ & 246 \\
\hline Lone vacancies & 0 & 250 & $\mathrm{~N} / \mathrm{A}$ & 250 \\
\hline Lone anti-sites & 8 & 19 & $\mathrm{~N} / \mathrm{A}$ & 27 \\
\hline 1NN di-vacancies & 0 & 1 & 0 & 1 \\
\hline 2NN di-vacancies & 0 & 2 & 0 & 2 \\
\hline Tri-vacancies & 0 & 1 & 0 & 1 \\
\hline 1NN di-Interstials & 0 & 11 & 0 & 11 \\
\hline 2NN di-Interstials & 0 & 2 & 0 & 2 \\
\hline Tri-interstials & 0 & 0 & 0 & 0 \\
\hline 1NN di-anti-sites & 0 & 0 & 95 & 95 \\
\hline 2NN di-anti-sites & 0 & 0 & 1 & 1 \\
\hline Tri-anti-Sites & 0 & 0 & 0 & 0 \\
\hline Anti-site+mono-vacancies & 0 & 2 & 0 & 2 \\
\hline Anti-site+mono-interstials & 2 & 0 & 0 & 2 \\
\hline Split-interstials & 0 & 1 & 1 & 2 \\
\hline Split-vacancies & 0 & 4 & 1 & 5 \\
\hline Vacancy-interstials & 0 & 12 & 0 & 12 \\
\hline Unclassified tri-defects & 0 & 3 & 16 & 19 \\
\hline
\end{tabular}

Table 1: Quantities of outlying defect clusters (outside the core region), from $5 \mathrm{keV}$ collision cascade simulations into $\mathrm{Pu} 5$ at. \% Ga. Values represent the total number of residual defects at 20 ps from 60 collision cascades. Note the small number of Ga vacancies or interstitials, yet the abundance of anti-site defects suggesting nearly all Ga displaced during the cascade finish occupying lattice sites. 


\begin{tabular}{|l|l|l|l|}
\hline \multicolumn{4}{|c|}{ Pure Pu } \\
\hline \multicolumn{4}{|c|}{ Peak } \\
\hline \hline & Time (fs) & Defects & Volume $\left(\mathrm{nm}^{3}\right)$ \\
\hline \hline Average & 1945 & 3117 & 179 \\
\hline Stan. Dev. & 300 & 650 & 28 \\
\hline \multicolumn{4}{|c|}{ Residual } \\
\hline \hline Average & 20000 & 1512 & 80 \\
\hline Stan. Dev. & 15 & 391 & 22 \\
\hline
\end{tabular}

\begin{tabular}{|l|l|l|l|}
\hline \multicolumn{4}{|c|}{ Pu 5 at. \% Ga } \\
\hline \multicolumn{4}{|c|}{ Peak } \\
\hline \hline & Time (fs) & Defects & Volume $\left(\mathrm{nm}^{3}\right)$ \\
\hline \hline Average & 2086 & 3886 & 205 \\
\hline Stan. Dev. & 136 & 514 & 15 \\
\hline \multicolumn{4}{|c|}{ Residual } \\
\hline \hline Average & 20000 & 1521 & 85 \\
\hline Stan. Dev. & 20 & 358 & 16 \\
\hline
\end{tabular}

\begin{tabular}{|l|l|l|l|}
\hline \multicolumn{4}{|c|}{ Percentage Change } \\
\hline \hline Peak & $7.3 \%$ & $24.7 \%$ & $14.4 \%$ \\
\hline Residual & $0 \%$ & $0.57 \%$ & $6.29 \%$ \\
\hline
\end{tabular}

Table 2: Analysis of the core region of disorder for all $5 \mathrm{keV}$ cascades in both pure $\mathrm{Pu}$ and $\mathrm{Pu} 5$ at. \% Ga. Indicates the increase in the number of defects at peak with the inclusion of the $5 \% \mathrm{Ga}$, which is not fully compensated by the increase in the core volume. 


$$
N_{f}=A\left(E_{P K A}\right)^{m}
$$

(where $N_{f}$ is the number of Frenkel pairs and $E_{P K A}$ is the PKA energy). This generates values of around 319 and 1.55 for $A$ and $m$ respectively (Figure 15). The key value $m$ is much larger than found in studies for other fcc metals, which are reported to have an average value of $m$ around 0.77. Figure 15 also shows the fit from pure Pu studies [12] which are almost coincident, again suggesting the introduced Ga have no quantitative effect on residual defects. It should be noted that data deviates from the Kinchin-Pease [24] and NRT [25] analytical models of radiation damage, in which damage is linearly dependent on PKA energy. This is attributed to the inability of these model to treat the amorphous region created as PKA energy increases.

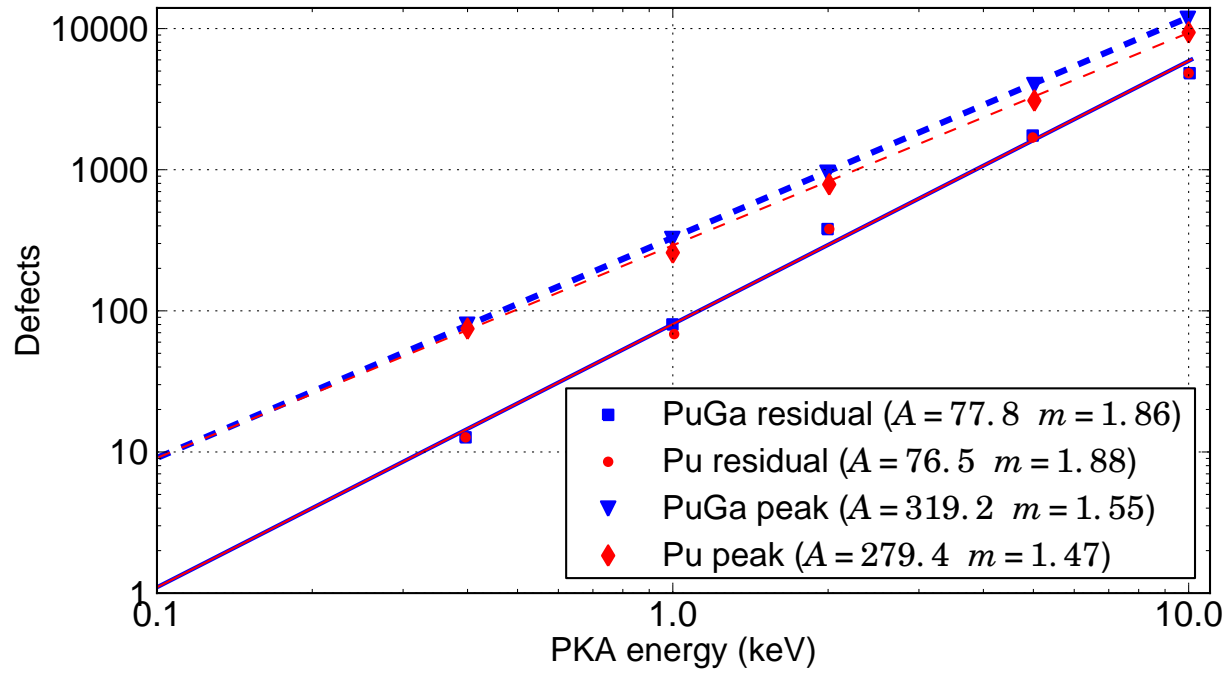

Figure 15: Residual and peak number of defects $\left(N_{f}\right)$ as a function of PKA energy $\left(E_{P K A}\right)$. Lines represent fits to the equation; $N_{f}=A\left(E_{P K A}\right)^{m}$, with constants $A$ and $m$ also shown. Pure Pu results are taken from previous work [12].

\section{Conclusion}

A comprehensive investigation has been carried out into radiation damage into Gastabilised fcc Pu. The study is centred around collision cascade simulations which incorpo- 
rated various $\mathrm{Pu} 5$ at. \% Ga configurations and sampled PKA energies in the range from 0.4 to $10 \mathrm{keV}$. This resulted in over 600 separate simulations allowing for detailed statistics and analysis. Threshold displacement energies $\left(E_{d}\right)$ were also calculated to give insight to the underlying cascade processes.

Calculations of $E_{d}$ suggested a wide range of values from $5 \mathrm{eV}$ to $40 \mathrm{eV}$ for the Pu PKA and from $8 \mathrm{eV}$ to $70 \mathrm{eV}$ for the Ga PKA, dependent on PKA trajectory and Ga arrangement. The minimum value of $E_{d}$ calculated for the $\mathrm{Pu} \mathrm{PKA}$ is similar to that found in pure $\mathrm{Pu}$ [12] at around $5 \mathrm{eV}$, which relates to impacts along directions of low atomic density in $\mathrm{Pu}$ rich regions.

Collision cascade results showed that the displaced Ga atoms returned to a local site within the ballistic phase resulting in few isolated Ga defects. Quantitatively, the residual defects produced after 20 ps showed no distinct differences to the pure $\mathrm{Pu}$ case[12], with the resultant cascade structure being similar. The results show a large variation in defect production dependent on the PKA direction and $\mathrm{Pu} 5$ at. \% Ga configuration. The number of residual defects showed a wide variance at the higher energies of 5 and $10 \mathrm{keV}$ due to the development of sub-cascades. As with previous studies into pure $\mathrm{Pu}$ [12], the number of defects remaining after the first 10 ps of the collision cascades into $\mathrm{Pu} 5$ at. \% Ga is larger than for other fcc metals and increases with energy at a faster rate. This relates to the formation of an amorphous zone at the cascade core, that was found to be of around $5 \mathrm{~nm}$ diameter at $5 \mathrm{keV}$ which supports previous findings [11, 13]. This feature seems to be readily produced due to the instability of the material and its possible decomposition into various thermodynamically close phases. These findings suggest that although the substitutional Ga does stabilise the fcc phase, its inclusion does not improve the radiation tolerance of plutonium.

\section{Acknowledgements}

The authors would like to thank Steve Valone for useful discussions. Acknowledgement is also given to EPSRC and the AWE for funding. 


\section{References}

[1] S. S. Hecker, Los Alamos Science pp. 290-335 (2000).

[2] M. I. Baskes, Physical Review B 62, 15532 (2000).

[3] A. Kubota, W. G. Wolfer, S. M. Valone, and M. I. Baskes, Journal of Computer-Aided Materials Design 14, 367 (2007).

[4] S. M. Valone, Journal of Nuclear Materials 324, 41 (2004).

[5] M. I. Baskes, A. C. Lawson, and S. M. Valone, Physical Review B 72, 1 (2005).

[6] R. S. Averback and T. Diaz De La Rubia, Solid State Physics 51, 281 (1997).

[7] L. Berlu, G. Jomard, G. Rosa, and P. Faure, Journal of Nuclear Materials 372, 171 (2008).

[8] B. P. Uberuaga, S. M. Valone, and M. I. Baskes, Journal of Alloys and Compounds 444-445, 314 (2007).

[9] B. P. Uberuaga and S. M. Valone, Journal of Nuclear Materials 375, 144 (2008).

[10] L. Berlu, G. Jomard, G. Rosa, and P. Faure, Journal of Nuclear Materials 374, 344 (2008).

[11] V. V. Dremov, F. A. Sapozhnikov, S. I. Samarin, D. G. Modestov, and N. E. Chizhkova, Journal of Alloys and Compounds 444-445, 197 (2007).

[12] M. Robinson, S. D. Kenny, R. Smith, M. T. Storr, and E. McGee, Nuclear Instruments and Methods in Physics Research Section B: Beam Interactions with Materials and Atoms 267, 2967 (2009).

[13] N. Baclet, B. Oudot, R. Grynszpan, L. Jolly, B. Ravat, P. Faure, L. Berlu, and G. Jomard, Journal of Alloys and Compounds 444-445, 305 (2007).

[14] M. I. Baskes, K. Muralidharan, M. Stan, S. M. Valone, and F. J. Cherne, JOM Journal of the Minerals, Metals and Materials Society 55, 41 (2003).

[15] M. I. Baskes, S. P. Chen, and F. J. Cherne, Physical Review B 66, 1 (2002).

[16] M. I. Baskes, S. Y. Hu, S. M. Valone, G. F. Wang, and A. C. Lawson, Journal of Computer-Aided Materials Design 14, 379 (2007).

[17] J. F. Ziegler, J. P. Biersack, and U. Littmark, The stopping and range of ions in matter (Pergamon, New York, 1985).

[18] R. M. Hestenes and E. Stiefel, Journal of Research of the National Bureau of Standards 46, 409 (1952).

[19] W. G. Hoover, Physical Review A 31, 1695 (1985).

[20] D. T. Lee and B. J. Schachter, International Journal of Parallel Programming 9, 219 (1980).

[21] F. P. Preparata and S. J. Hong, Comm. ACM 20, 87 (1977).

[22] M. W. Guinan and J. H. Kinney, Journal of Nuclear Materials 104, 1319 (1981).

[23] T. Diaz De La Rubia, R. S. Averback, R. Benedek, and W. E. King, Physical Review Letters 59, 1930 (1987).

[24] G. H. Kinchin and R. S. Pease, Reports on Progress in Physics 18, 1 (1955).

[25] M. J. Norgett, M. T. Robinson, and I. M. Torrens, Nucl. Eng. Des 33, 50 (1975). 